\title{
Germanica
}

\section{Retours contemporains sur une jeunesse catholique et romaine : rancœur et étonnement}

\section{Anky Hilgersom et J.M.J. Sicking}

Traducteur : Henri-Philippe Faucher

\section{OpenEdition \\ Journals}

Édition électronique

URL : http://journals.openedition.org/germanica/1939

DOI : $10.4000 /$ germanica. 1939

ISSN : 2107-0784

\section{Éditeur}

Université de Lille

\section{Édition imprimée}

Date de publication : 31 décembre 1996

Pagination : $33-47$

ISSN : 0984-2632

\section{Référence électronique}

Anky Hilgersom et J.M.J. Sicking, « Retours contemporains sur une jeunesse catholique et romaine : rancœur et étonnement », Germanica [En ligne], 19 | 1996, mis en ligne le 04 juin 2013, consulté le 06 octobre 2020. URL : http://journals.openedition.org/germanica/1939 ; DOI : https://doi.org/10.4000/ germanica.1939

Ce document a été généré automatiquement le 6 octobre 2020.

(c) Tous droits réservés 


\title{
Retours contemporains sur une jeunesse catholique et romaine : rancœur et étonnement
}

\author{
Anky Hilgersom et J.M.J. Sicking \\ Traduction : Henri-Philippe Faucher
}

\section{Les riches heures de la vie catholique et romaine}

1 L'hebdomadaire familial Katholieke Illustratie publia, de 1926 à 1932, une chronique photographique intitulée Uit het rijke Roomsche Leven (Les Riches heures de la vie romaine), consacrée à toutes sortes d'événements mémorables ayant eu lieu dans les milieux catholiques. Ordinations de prêtres, anniversaires d'ecclésiastiques, noces d'or, réunions commémoratives d'associations, distinctions pontificales, bénédictions d'écoles et d'autres bâtiments, visites épiscopales, départs des missionnaires pour l'étranger -, tous les détails de la vie de la grande famille catholique aux Pays-Bas furent réunis avec application.

Uit het rijke Roomsche Leven (Les Riches heures de la vie romaine) est aussi le titre d'un récit documentaire richement illustré publié par Michel van der Pias en 1927 et portant sur une période de l'histoire des Pays-Bas catholiques qui atteignit son apogée dans l'entredeux-guerres et se poursuivit encore après, pendant une période assez longue. À cette époque, la vie politique, sociale et culturelle était fortement "compartimentée " ("verzuild»), ce qui signifiait que les différentes classes de la population s'étaient organisées sur la base de convictions religieuses ou éthiques, et qu'elles essayaient continuellement de préserver leur propre identité dans une concurrence réciproque. Ce phénomène s'appliquait notamment aux catholiques, aux protestants et aux socialistes, qui, eux, se distinguaient de ceux qui étaient «neutres » ou « libéraux » et qui ne formaient pas de groupe bien défini.

$3 \mathrm{Au}$ sein de leur groupement religieux, les catholiques, qui se considéraient comme «faisant partie de la maison de Dieu», menaient une vie de confiance et de combat. 
Fidèles du pape qui aimaient se considérer comme catholiques romains ou encore de préférence romains, ils chantèrent de bon cœur dans leurs réunions le chant suivant :

Romains nous sommes au fond de notre cœur et de notre âme

Romains nous sommes par le geste et par la parole

Romains, malgré les affronts de l'infortune et de la souffrance

Romains jusqu'à ce que sonne l'heure de la mort.

Romains en notre demeure, romains aussi au dehors

N'ayons jamais honte de notre choix honorable ;

$\mathrm{Ni}$ la lâcheté ni la peur ne nous feront taire ;

Romains, nous le sommes, c'est notre devise.

Aucun groupe n'avait mis en place une organisation aussi étendue que celle des catholiques. Ils étaient représentés par des «conseillers spirituels " à peu près à tous les niveaux. Ils avaient leur propre parti politique, leurs propres organisations professionnelles et leurs propres syndicats, leur propre société de radio et leurs propres journaux écoles et université, leurs propres hôpitaux et maisons de retraite, leurs propres maisons d'édition et leurs propres fanfares mouvements de jeunesse et de femmes, leurs propres associations sportives et écoles de danse, leurs propres associations d'étudiants, des bibliothèques, etc. Les films étaient de préférence soumis à une censure supplémentaire, un service d'information fixait les livres que l'on pouvait lire par tranche d'âge ; on déplorait et l'on combattait le plus possible les mariages avec les «hétérodoxes ». L'influence de l'Église était visible et perceptible à tous les niveaux dans les milieux catholiques. Ce n'est pas pour rien qu'une exposition, organisée récemment à Utrecht autour des riches heures du catholicisme de 1900 à 1950, fut intitulée « Catholique en Tout».

5 Examinons de plus près le monde catholique de cette époque. Vers la fin des années soixante-dix, la K.R.O. [= Société de radiodiffusion catholique] diffusa à la télévision une série très suivie désirant donner un aperçu réaliste mais quelque peu idéalisé de la question. L'histoire, intitulée Dagboek van een herder shond (Journal d'un chien berger), et qui fut publiée par la suite sous forme de livre, raconte les aventures d'un jeune vicaire de paroisse dans un village du Limbourg. Ce vicaire est la maladresse en personne et a fort à faire avec le curé et les autres autorités du village, mais il arrive toujours à mener les choses à bonne fin, car il a le cœur bien placé. Les apparences de la vie spirituelle, avec son culte solennel ses processions pittoresques et ses rituels immuables autour de la naissance le mariage et la mort, forment un décor omniprésent. Tandis qu'Erik Odekerke s'affaire à régler des conflits en tout genre et à veiller au maintien de la morale conjugale, il converse souvent de façon intime avec un collègue d'un village voisin comme avec son ange gardien. Cela lui permet d'avoir prise sur les événements et d'avoir confiance, même s'il doit lui-même lutter contre les tentations de l'amour charnel et qu'il est touché par les questions sociales importantes dans la région minière.

$6 \quad$ La série télévisée Dagboek van een herdershond (Journal d'un berger), qui remporta un tel succès que l'on en fit une suite, était une adaptation de la première partie de la Kroniek eener parochie (Chronique d'une paroisse) (1941-1948) du père Jacques Scheurs. Willy van Hemert, le réalisateur, avait pris des libertés en adaptant cet ouvrage, notamment en plaçant les questions se rapportant à la mine à l'arrière-plan, et les tribulations sentimentales du jeune vicaire au premier. C'est ainsi que de nombreux téléspectateurs eurent en partie l'impression qu'ils ne regardaient pas une histoire se déroulant environ à l'époque de la Première Guerre mondiale, mais un passé à peine révolu. $\mathrm{Ce}$ 
n'était pas étonnant. La Deuxième Guerre mondiale n'a jamais eu, en effet, qu'une valeur relative comme coupure dans l'histoire sociale et culturelle des Pays-Bas. Ce n'est que vers la fin des années soixante, à la suite de la révolte étudiante à Paris et du mouvement provo à Amsterdam, que les normes et les valeurs traditionnelles furent ébranlées sur une grande échelle et que le compartimentage commença à appartenir également, petit à petit, au passé. Ce n'est qu'à partir de ce moment-là que la vie « catholique féconde » eut à envisager sa perte.

\section{Retours littéraires sur une jeunesse catholique}

7 En 1954, les évêques néerlandais avaient encore essayé par un écrit pastoral d'empêcher les catholiques de se joindre à des organisations non-catholiques, mais le processus de "décompartimentage " devait s'avérer finalement impossible à enrayer ; c'est ce que montrent clairement, par exemple, les défaites électorales que devaient subir les partis confessionnels à partir de 1967. Alors que les rénovations que le concile Vatican II (1962-1965) avait également accomplies aux Pays-Bas allaient trop loin pour les «conservateurs " et justement pas assez loin pour les "progressistes ", l'Église perdit rapidement de son autorité ; la sévère morale conjugale se relâcha, les prêtres et les religieux furent nombreux à enfreindre ouvertement les règles du célibat, et la laïcité se développa même dans les campagnes. Ce furent surtout les jeunes qui prêtèrent l'oreille au désir de liberté et de démocratisation qui se répandait et qui s'opposèrent à l'autorité officielle. L'éducation anti-autoritaire commença à se répandre, les efforts pour aboutir à l'épanouissement personnel exigèrent de plus en plus de liberté de choix et de mouvement, l'introduction de la pilule anticonceptionnelle facilita plus que jamais la mise en pratique d'une morale sexuelle libre. Le slogan «l'imagination au pouvoir " traduisait un élan nouveau; une façon de sentir et de penser plus large semblait devoir mettre un terme à la mentalité bourgeoise que l'on ressentait comme étouffante et bornée.

Dans les années soixante et soixante-dix, des écrivains comme Jan Wolkers et Maarten't Hart devinrent célèbres et firent scandale en publiant des romans et des récits qui en finissaient une fois pour toutes avec l'éducation calviniste austère. À partir des années 1980, des écrivains d'origine catholique se mirent à passer en revue leur jeunesse de façon critique. La plupart d'entre eux étaient nés dans les années 1940-1950 et appartenaient de ce fait à la génération élevée dans un milieu catholique traditionnel, mais s'en étaient finalement arrachés. Jeroen Brouwers (1940), Boudewijn Büch (1948), Nicolette Smabers (1948), A.F.Th. van der Heijden (1951), Huub Beurskens (1950) et Ton van Reen (1941) furent les écrivains les plus connus; Johan Buschman (1924), Noor Smals (1936) et Jasper Mikkers (1948, dont les premiers ouvrages parurent sous le pseudonyme de Tymen Trolski), des auteurs moins connus, s'ajoutèrent à cette liste ultérieurement. Leurs œuvres - du reste à aucun égard celles d'un groupe - présentent de façon étonnante nombre d'analogies par la façon dont les protagonistes avaient fait autrefois l'expérience du catholicisme. Ce thème ressort davantage dans certains ouvrages que dans d'autres et le contexte donné peut différer passablement, mais leurs ouvrages sont presque toujours un traitement littéraire de données autobiographiques facilement reconnaissables. 


\section{Discipline, obédience et châtiment}

9 Dans le roman Ingetuigd en ingetogen (Harnachée et rangée) (1995) de Noor Smals, une jeune fille du nom de Monica Donker se retrouve avec sa cousine, vers 1950, dans un pensionnat de jeunes filles dirigé par les Chanoinesses de Saint Augustin. Dans une brochure, les élèves sont comparées à des « pur-sang » dont on doit former le caractère par la « discipline et l'entraînement ». Les chanoinesses attachent une grande valeur à la maxime finement brodée «Je ferai ce que tu commanderas, commande ce que tu veux ». Monica a tendance à révérer la pensée de cette maxime, mais son amie réfractaire Gertie y reconnaît un symptôme de la structure du pouvoir à laquelle elles sont toutes confrontées. La Mère Préfète, qui fait la pluie et le beau temps pour les élèves, s'incline elle-même devant la Révérende Mère ; cette supérieure doit à son tour s'incliner devant l'autorité du pape, qui se trouve lui-même placé sous les ordres de Dieu.

10 La présence d'autorités qui ne tolèrent aucune discussion et qui déterminent tout simplement les règles et les normes, était chose tout à fait normale dans la société d'avant 1970 ; cela se produisait également au travail, à l'école et au sein de la famille. Mais celui qui mettait en doute les conceptions régnantes dans un milieu catholique croyant et se heurtait alors à l'autorité, se retrouvait confronté à tout un système, qui en appelait en dernier ressort à la volonté de Dieu en personne. Se révolter contre cette volonté faisait de vous, par définition, quelqu'un de mauvais, un pécheur, qu'il s'agissait de faire fléchir.

11 Ce sujet est décrit à plusieurs reprises avec toutes sortes de nuances dans les romanssouvenirs placés dans un contexte catholique. Ce que Monica Donker et ses amies vivent chez les nonnes n'est rien comparé aux expériences que Jeroen Brouwers et Boudewijn Büch ont intégrées à leurs livres. Brouwers, qui est né dans les anciennes Indes orientales et qui se retrouva dans un camp d'internement japonais avec sa mère et sa sœur, a déclaré à plusieurs reprises que sa personnalité a été à jamais endommagée pendant la période d'après-guerre qu'il passa dans un pensionnat catholique aux Pays-Bas. Il a ainsi déclaré dans une interview :

Mon véritable traumatisme, je ne l'ai pas subi dans les camps japonais, mais pendant ces années de pensionnat. C'est à ce moment-là que l'on a détruit à jamais quelque chose à l'intérieur de moi-même. Le dressage catholique : dans ces écoles, l'éducation consistait en asservissement et terreur morale. (Journal Trouw, 2-41987).

Les expériences de jeunesse de Brouwers - voir aussi son essai autobiographique dans De Bierkaai. Kladboek 2 (Sans espoir de victoire. Cahier de brouillon 2) - se retrouvent également dans sa trilogie dite des Indes. Le caractère particulier de livres comme Bezonken rood (Rouge décanté) (1981) et De zondvloed (Le Déluge) (1988) est qu'ils ne prétendent pas former une recherche du passé, mais qu'ils veulent surtout montrer comment toutes sortes d'événements appartenant au passé se sont liés entre eux et jouent ensemble en permanence un rôle actif dans le présent. Un exemple important de ce phénomène nous est donné par le fait que le personnage principal de De zondvloed établit souvent une relation entre l'angoisse, qui le tient à sa merci même à un âge avancé, et les expériences faites étant enfant. En outre, les frères qui mettent leur nez partout à l'école - «malades et possédés, et avides de surprendre et de punir » ressemblent aux anciens gardiens du camp. 
13 Boudewijn Büch, qui fut placé vers l'âge de dix ans dans un hôpital psychiatrique catholique pour enfants, car son entourage avait peur qu'il entretienne des relations à tendance sexuelle avec son père, ne s'est pas exprimé moins violemment sur de telles pratiques que Brouwers. Il ressort de différentes interviews qu'il considérait que le catholicisme s'apparentait au fascisme, même s'il voulait bien admettre que la psychiatrie d'autrefois ne valait rien sur toute la ligne.

Het Dolhuis (La Maison de fous) (1987) est l'un des romans dans lesquels Büch décrit violemment son traumatisme de jeunesse. La façon dont le petit Winckler Brockhaus est accueilli dans ce livre à son arrivée à la "Maison des enfants bienheureux », est caractéristique de la suite des événements. Le fait que le garçon annonce à la nonne de service qu'il n'est pas malade mais seulement quelque peu nerveux, conduit à l'altercation

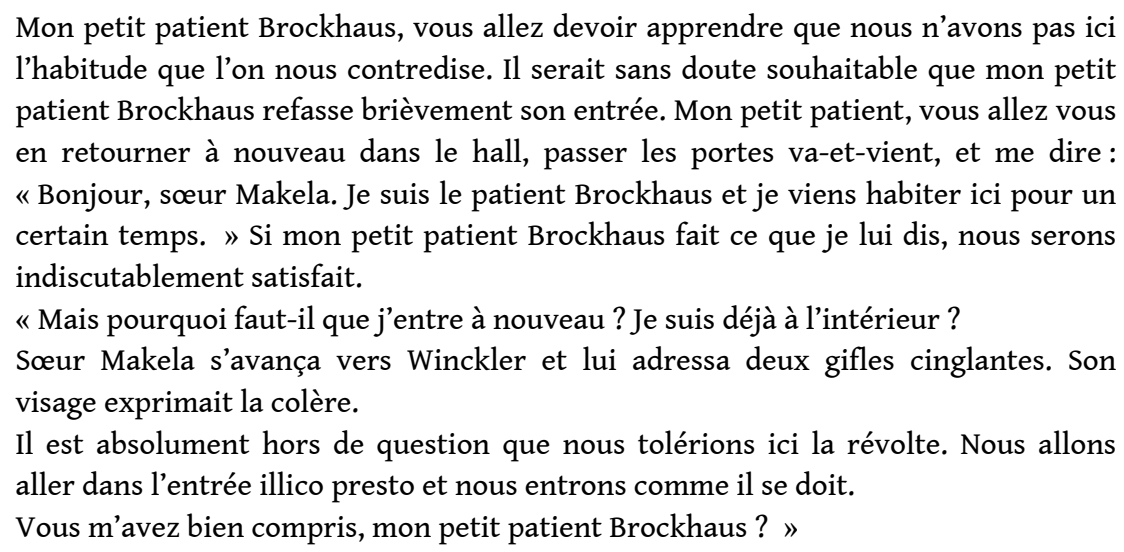

$15 \mathrm{Au}$ cours du traitement dont le but est de "chasser toute la saleté » et de «laver sa petite âme de pécheur ", Winkler devra subir bon nombre de châtiments corporels et de vexations. Il en gardera le sentiment qu'il ne pourra plus jamais se réconcilier avec lui-même ni avec la vie.

\section{Contrition et sentiment de culpabilité}

Dans le roman De Paarse Stempel (Le Sceau violet) (1990) dont l'action se déroule dans un pensionnat catholique des années trente, le jeune Bennie se demande désespérément ce qui finalement n'est pas défendu. Lui-même et tous ses compagnons d'infortune plus âgés doivent en effet se plier à une série de prescriptions et de règles dont la violation est sévèrement punie. En y regardant de plus près, le but poursuivi est souvent de réprimer toute forme de caractère et d'individualisme. Et c'est justement sur ce point que tous les protagonistes des romans dont nous traitons ici se distinguent de leur entourage.

On inculque souvent aux "pécheurs " réels comme aux "pécheurs " potentiels l'idée que Dieu voit tout et que l'on ne se moque pas de lui impunément. Les sentiments de culpabilité et d'angoisse ne tardent donc pas à se manifester, notamment lorsqu'une sexualité naissante est en jeu. Le contraste violent entre l'amour spirituel soi-disant noble et les désirs charnels que l'on considère comme vils, existait encore vers 1950 dans bon nombre de cercles confessionnels. Presque tous les romans-souvenirs sur fond de catholicisme décrivent la manière dont les éducateurs étaient hantés par la crainte que les enfants en grandissant ne pèchent contre la chasteté. 
Cette morale traditionnelle est exprimée dans le roman de Jeroen Brouwers De zondvloed, lorsque le père préfet commence à faire à haute voix la lecture au personnage principal.

Cela traite de "chasteté ", qui est "la vertu qui me permet d'être maître des impulsions les plus fortes de ma nature humaine : les désirs de mon corps ", et du dessein formé par Dieu concernant ces impulsions les plus fortes de ma nature humaine : «que celles-ci ne sont utilisées que dans la mesure où Dieu a élu l'homme à participer à la Création, au maintien du genre humain, donc dans le mariage »; il est souvent question de " la mort », de " châtiments terribles », de « l'enfer ».

Le personnage principal pense un peu plus loin dans le livre :

Toujours le péché, toujours le châtiment. Toute la journée, pratiquement sans interruption, les pensées envahies uniquement par cette seule chose, toujours la même : la partie du corps interdite. Chasteté, morale, règles, contrôle, discipline. Le sixième et le neuvième commandement. [...] Toujours se confesser, cet étranglement, les cheveux courts, toujours cette eau glacée qui s'écoule sur moi en guise de châtiment. Le bruit de cette eau qui coule.

Le sentiment de culpabilité et de contrition en matière de sexualité apparait également dans les romans-souvenirs où le personnage principal ne reçoit pas une éducation catholique sévère, comme dans la première partie du grand cycle de A.F. Th. van der Heyden De tandeloze tijd (Le Temps sans outrage). Le jeune Albert Egberts de De gevarendriehoek (Le Triangle du danger) (1985) n'est pas le seul à penser que ses " cochonneries" sont la cause des événements catastrophiques qui se déroulent dans son entourage direct.

On décrit souvent également comment les jeunes enfants sont déséquilibrés lors de la préparation à la " première communion ", lorsqu'il sont obligés de faire connaissance avec la notion de "péché ", qui peut conduire aux châtiments les plus horribles non seulement pendant la vie sur terre mais aussi dans l'« au-delà ». Ce qui a surtout laissé une impression indélébile, c'est le livre d'images que l'on utilisait beaucoup autrefois et dans lequel on avait représenté de façon imagée comment une âme pure et blanche pouvait se souiller de tâches d'encre noire par toutes sortes de méfaits, de telle sorte que le visage du méchant diable se couvrait de grimaces perfides et que l'ange gardien se détournait d'un air affligé.

Les conséquences de tous ces péchés, dont les noms étaient en grande partie confus, étaient souvent assez vagues, mais c'est justement pour cela qu'elles pouvaient susciter facilement chez les enfants toutes sortes d'angoisses et de questions singulières. Les récits de Nicolette Smabers, dont l'action se déroule dans les milieux catholiques haguois pendant les années cinquante et soixante, fournissent des exemples éloquents à ce sujet. Lorsque la jeune fille du recueil De Franse tuin (Le Jardin français) (1983), près d'une chaufferie terrifiante avec toutes sortes de tuyaux bizarres, marche avec précaution sur des morceaux de verre dépoli, elle se met à penser subitement :

Pénétrer dans un domaine : marcher avec une grande précaution sur les dalles en verre et celui qui est mauvais passe à travers.

Elle se demande à plusieurs reprises si les infidèles et les bandits n'éprouvent pas plus de plaisir que les honnêtes catholiques, parce que attendre bien sagement la fin de la messe tous les dimanches à l'église avec une "âme blanchie " n'est pas non plus finalement très palpitant. Et ne vaut-il pas mieux se réjouir immédiatement de tous ses péchés plutôt que de nourrir l'espoir d'un au-delà ennuyeux et abstrait? 
Etre assis pour toujours sur une chaise et contempler Dieu et la vierge Marie. Dieu et la vierge Marie de leur côté ne faisaient rien d'extraordinaire ; un couple célébrant ses noces d'or, des habits cousus d'or, une couronne, des anges et de la crème Chantilly tout autour. Cela m'oppresse, mourir et attendre éternellement. plusieurs reprises par les prêtres qui prêchent la chasteté, sont la preuve d'une
curiosité malsaine et admettent toutes sortes de privautés.

\section{Office rituel et magie}

De nombreux livres-souvenirs donnent l'impression qu'au sein de l'Église catholique on mettait davantage l'accent sur les règles de conduite que sur la conscience religieuse. Assister à la messe le dimanche, communier à jeun, manger du poisson à la place de viande le vendredi, ne pas se découvrir pendant les services religieux lorsqu'on est une jeune fille, garder les yeux bien fermés pendant la prière - ce genre de choses semblait revêtir une grande importance.

Lors des fêtes, les apparences extérieures étaient très importantes. Ainsi, le personnage principal du recueil de nouvelles de Nicolette Smabers, Portret van mijn engel (Portrait de mon ange) (1987) se rappelle très bien les préparatifs de la fête de sa première communion.

$\mathrm{Au}$ cours de la dernière semaine, on dut répéter à l'église. On s'exerçait à s'agenouiller les genoux serrés, à sortir la langue pour l'hostie les yeux fermés - ni trop loin, ni trop pointue, mais large, comme un bol, un bol que l'on offre. Et les paumes des mains jointes. Non, ne pas se pencher vers le sol comme sur un plongeoir. Et l'on s'entrânait avec les yeux mi-clos - mi-clos et non pas fermés -, retourner à sa place, ni trop rapidement, ni trop lentement, ne doubler personne, ne rien dire, ne pas chuchoter non plus, ne pas mâcher l'hostie ; si elle, elle n'est pas encore partie, elle fondra.

Rien ne nous initiait au mystère auquel nous devions participer sous peu. Ce dont il s'agissait se trouvait manifestement enfermé dans la précision déférente avec laquelle nous exécutions ces actes ; une chose qui serait appelée et qui apparaitrait le jour même de la fête. C'est en partie au moyen de la grâce qui se déversait à ce moment-là que vous deveniez, à cet instant sacré, un enfant de Dieu. La foi n'était peut-être qu'une question de légèreté.

La prière semblait aussi n'être souvent qu'une sorte de rituel. Dans Bezonken rood, Jeroen Brouwers fait dire par exemple à son alter ego :

Je n'ai jamais su ce que c'était vraiment que de prier. Il fut pourtant un temps où je connaissais par cœur toutes les prières, toutes les litanies et les formules de la religion catholique. Je m'aperçus assez rapidement que le seul fait d'énoncer ces textes n'était pas « prier », mais je ne comprenais pas ce qu'il manquait -,j'ai arrêté de me poser la question.

J'ai récité des millions d'Ave Maria, mais je n'ai pas prié un seul Ave Maria.

La formation à l'office pouvait avoir une signification plus profonde lorsqu'on lui attribuait une sorte de pouvoir magique. Ton van Reen, qui décrit dans sa série de livres commencée en 1986 comment un jeune villageois du Limbourg devient un homme adulte, montre de façon très précise les faits et gestes des catholiques dévots. Sa mère, qui ne fait pas une distinction très nette entre les anciennes formes de superstition et les coutumes de la religion catholique, a continuellement à faire aux exorcismes dans Ret Winterjaar (L'Hiver) (1986). 
Pour éloigner Satan de ma personne, elle épinglait chaque jour sur mon maillot de corps une rangée de médailles de la Sainte Vierge. Elle cachait des images de saints sous mon oreiller et faisait au crochet des petites croix presque invisibles sur mes vêtements.

Pour chasser le diable qui avait élu domicile dans mon corps, mère m'obligeait à de nombreuses prières. Je devais faire le signe de croix plusieurs fois par jour. Porter sur moi un rosaire. Tenir le catéchisme à porté de main. Communier quotidiennement. Honorer mon ange gardien.

L'appel de la mère aux vertus miraculeuses des saints, tous spécialisés, fait pendant à sa peur du diable. Ils peuvent être disposés favorablement, non seulement par le fait de faire brûler des cierges ou de placer des fleurs, mais aussi parce que l'on effectue des pélerinages. Le culte des images, des reliques, des médailles et d'autres objets particuliers, fait partie de l'arsenal du souvenir de tous les écrivains d'origine catholique. Une nouvelle du recueil de Huub Beurskens, Slapende hazen (Les Lièvres endormis) (1985), nous apprend comment il fallait s'y prendre pour renouveler soimême la provision d'eau bénite en cas d'urgence. Un jeune garçon maintient ses doigts mouillés à l'église dans sa bouche de façon crispée et sans avaler, de sorte qu'une fois à la maison, il peut transformer avec sa salive de l'eau ordinaire en la "plus pure des eaux bénites qui soit ». La foudre ne tomba pas cette année-là, personne ne fut malade et les saisons suivirent leur cours comme à l'accoutumée.

\section{Aliénation et éloignement}

Dans l'une de ses nouvelles-souvenirs, Nicolette Smabers écrit: «les affirmations m'entouraient comme les points d'un cercle». Mais ces affirmations inébranlables basées sur la foi éveillaient justement en la jeune fille grandissante des doutes et suscitaient des questions. C'est en vain qu'elle s'interroge sur des mots incompréhensibles qui formulent des affirmations tout aussi incompréhensibles: la miséricorde de Dieu et son ubiquité, le corps glorifié du Christ, la virginité de la Sainte Vierge. Et elle n'est pas satisfaite lorsque la sœur lui rappelle qu'elle doit croire " par la voie cœur» ce qu'elle ne peut concevoir par la raison. En même temps, elle est convaincue que la foi n'apporte rien dans les faits.

Dieu ne se manifestait pas vraiment. Mais il fallait prier et croire, de même qu'il ne fallait pas parler la bouche pleine et qu'il fallait se satisfaire de ce que l'on recevait, tout comme il fallait faire des additions et apprendre à lire. (Portret van mijn engel [Portrait de mon ange], 1987).

31 Aussi la crise ne tarde-t-elle pas. Pendant la communion solennelle suivie de la profession de foi, le personnage principal s'exclame subitement :

Je ne crois pas en toutes ces choses de l'Église. Je n'y comprends rien du tout, tout simplement. Je n'ai jamais rien pigé.

Le processus qui conduit à se détacher de la foi dans laquelle on a été élevé est souvent progressif. Dans son recueil d'essais Buitenwegen (Des chemins détournés) (1992), Huub Beurskens décrit le modèle courant: les problèmes posés par l'accomplissement de la pratique dominicale en sont le symptôme. Un enfant commence en grandissant à se plaindre de l'obligation d'assister à la sainte messe dominicale. Au début, on se tire encore d'affaire en allant officiellement à l'église et en faisant autre chose pendant la messe. Mais tôt ou tard, le moment arrive où l'on annonce ouvertement à la maison que l'on n'ira plus à l'église ; les conflits qui en résultent doivent alors suivre leur cours. 
'est chez ceux qui ont l'impression de souffrir véritablement d'une autorité fortement marquée par la religion que les sentiments de révolte sont évidemment les plus forts. Dans Het Dolhuis (La Maison de fous) - paru en 1987 et réédité déjà pour la quatorzième fois en 1992 - Boudewijn Büch décrit l'amertume ressentie par Winkler Brockhaus d'avoir à louer, pendant la messe obligatoire, « un Dieu qui ne s'est pas adressé à lui, un Dieu même qu'il méprisait ». Et dans De blauwe salon (Le Salon bleu) (1981), le personnage principal, qui ressemble de façon étonnante au jeune Winkler, se propose bientôt, au cours de ses internements solitaires, de renier sa foi et de ne plus jamais accepter une quelconque autorite. Lorsqu'il revoit plus tard l'hôpital psychiatrique, transformé en maison de retraite pour personnes séniles, il se demande :

Se peut-il qu'une maison qui m'a fait subir tant d'atrocités se soit transformée en une forteresse de sécurité et de tendresse? Pourquoi [...] ai-je été harcelé et défiguré en ces mêmes lieux, alors que j'étais un petit garçon? Se peut-il qu'il y règne un état d'affection et de sollicitude? Pourquoi a-t-il fallu que, garçon, je sois victime d'un régime religieux, expert en psychologie de l'âme?

Si Jeroen Brouwers est encore plus irréconciliable dans Het verzonkene (Le Noyé) (1979), c'est en partie à partir de son rôle en tant qu'écrivain.

Jamais plus, je ne veux jamais plus voir chez moi, pour finir, les monstres harpies et vindicatifs de la morale et de la religion. Je ne veux plus voir chez moi ce bétail pieux aveuglé de Laban, qui vient me raconter que j'aurais reçu mes talents de Dieu en personne, et que Lui m'a élu pour propager la Parole divine au moyen du Verbe et de la Langue. [...] Pointons d'abord nos armes à feu sur Dieu. Que tout d'abord la Parole de cet individu soit effacée de la mémoire de tous. Personne ne m'a donné le moindre talent. Dieu c'est moi, le Créateur c'est moi, le destructeur c'est moi. Amenez-vous en souriant avec de l'encens, amenez-vous avec de jolies filles, amenez-vous avec des animaux dociles, amenez-vous avec des fruits, amenez-vous avec un bon caractère et de la bienveillance. Je vous montrerai le cadavre du Christ, rien ne m'empoisonne autant l'existence, aujourd'hui encore, que Dieu.

\section{Conclusion}

On aura sans doute remarqué que les romans et les nouvelles contemporains où figurent de nombreux retours sur une jeunesse catholique et romaine, ne sont pas nés d'un sentiment de mélancolie. Les personnages principaux, qui, sur des points essentiels, renvoient clairement aux auteurs eux-mêmes, se sont tous révoltés, jeunes, contre une expérience de la foi qui engendra chez eux de plus en plus de questions et de plus en plus de résistance, et qui semblait n'avoir pas grand chose en commun avec un sentiment religieux. Ils se sentaient isolés dans leur révolte. Ils avaient à combattre la puissance des autorités, et leurs compagnons d'infortune, qui acceptaient en général les choses comme elles étaient, ne leur apportèrent pas un grand soutien. Dans de nombreux retours en arrière, la rancœur est encore manifestement présente, parfois mêlée d'étonnement pour des pratiques qui semblent revêtir aujourd'hui un aspect presque folklorique.

L'image des derniers chapitres de l'histoire des « riches heures catholiques » esquissée ces dernières décennies dans la littérature néerlandaise, fait partie à plusieurs égards de l'image beaucoup plus générale d'une époque. Il est évident que la vision des écrivains, nés en majeure partie vers 1950, a été en partie déterminée par les pensées révolutionnaires qui bouleversèrent les esprits vers 1970. Le sentiment de liberté était au premier plan. Et lorsqu'un poète comme Huub Beurskens développe à nouveau, dans 
un recueil récent, les grandes questions posées par la vie et la mort, ce n'est pas pour rien qu'il écrit les vers suivants :

Ne me faites pas de sermon sur un seul dogme, sur un seul Dieu, sur un corps et sur une âme. Laissez-moi non-converti, totalement désinvolte, créature nue créature nue sur un cheval gris pommelé sans selle qui va au pas dans le murmure de l'eau qui coule.

\section{RÉSUMÉS}

Le compartimentage (verzuiling) de la vie politique, sociale et culturelle fut à son apogée durant la période de l'entre-deux-guerres. Les différentes classes de la population s'étaient regroupées sur la base de convictions philosophiques ou religieuses et s'efforçaient constamment de préserver leur identité vis-à-vis des autres groupes. Les catholiques, qui aimaient encore à s'appeler « romains » à cette époque, organisaient leur vie avec assurance et combativité. L'influence de l'église y était partout présente et manifeste. Après la deuxième guerre mondiale, les « riches heures romaines " n'étaient pas encore révolues. Les valeurs et les normes traditionnelles ne furent remises en question qu'à la fin des années soixante, au moment de la révolte estudiantine à Paris et du mouvement Provo à Amsterdam. Les cloisons divisant la société néerlandaise commencèrent à disparaître.

Après que des auteurs connus tels que Jan Wolkers et Maarten't Hart ait réglé leurs comptes avec une éducation calviniste rigoureuse, les auteurs d'origine catholique se mirent également à écrire romans et récits qui abordent de manière critique leur jeunesse « romaine ». Parmi eux on trouve Jeroen Brouwers, Boudewijn Büch, Nicolette Smabers, A.F. Th. van der Heijden, Huub Beurskens et Ton van Reen; la plupart sont nés vers 1950. On ne trouve aucune trace de nostalgie dans leurs œuvres. La vie catholique traditionnelle de cette époque éveille essentiellement rancœur et étonnement. Certains points sont récurrents. Parmi ceux-ci on retrouve surtout l'exigence d'obéissance liée à la discipline et aux punitions, l'accent mis sur un sentiment très fort de péché et de culpabilité, une attitude crispée en matière de sexualité, une prééminence de la forme et une inclination à privilégier la magie au détriment d'un sentiment religieux plus profond. Les personnages des romans deviennent finalement tous étrangers au catholicisme et prennent leurs distances.

L'image que les auteurs traités donnent de leur jeunesse fait partie d'un moment historique plus large. Leur attitude ne peut être séparée des idées révolutionnaires qui ont agité les esprits vers 1970. L'aspiration à la liberté et au développement personnel y occupe une place centrale.

Tegen het einde van de jaren zestig werden de traditionele normen en waarden op ruime schaal doorbroken, in aansluiting op de studentenopstand in Parijs en de provobeweging in Amsterdam. Toen begonnen ook de oude scheidingsmuren te verdwijnen.

Nadat bekende auteurs als Jan Wolkers en Maarten't Hart waren gaan afrekenen met hun strengcalvinistische opvoeding, gingen vanaf \pm 1980 ook vele van huis uit katholieke schrijvers in romans en verhalen kritisch terugblikken op hun «roomse» jeugd. Tot hen behoorden Jeroen Brouwers, Boudewijn Büch, Nicolette Smabers, A.F.Th. van der Heijden, Huub Beurskens en Ton van Reen, meestal geboren rond 1950. In hun werk ontbreekt een toon van weemoed. Het traditionele katholieke leven van vroeger wordt hoofdzakelijk bezien met gevoelens van wrok en 
verwondering. Enkele punten keren daarbij vaak terug. Daarbij gaat het vooral om de eis van gehoorzaamheid in samenhang met een regime van tucht en straf, een sterk ontwikkeld zondeen schuldbesef en een krampachtige houding ten opzichte van de seksualiteit, het overheersen van uiterlijke vormen en een neiging tot magie boven een dieper religieus besef. De verhaalfiguren raken tenslotte allemaal vervreemd van het katholicisme en distantiëren zieh daarvan.

Het beeld dat de besproken schrijvers van hun jeugd geven, maakt deel uit van een veel algemener tijdsbeeld. Hun houding daartegenover kan niet losgezien worden van de revolutionaire gedachten die rond 1970 de gemoederen in beroering zijn gaan brengen. Een verlangen naar vrijheid en persoonlijke ontplooiing stond daarbij voorop.

\section{AUTEURS}

ANKY HILGERSOM ET J.M.J. SICKING

Université de Groningue 